\title{
Evaluation of the Frequency of Suicidal Thoughts and Attempts and Related Factors in Female Adolescents with Anorexia Nervosa
}

\author{
İlknur Ucuz ${ }^{1}$, Ayla Uzun Çiçek ${ }^{2}$ \\ ${ }^{1}$ Inonu University Faculty of Medicine, Department of Child and Adolescent Psychiatry, Malatya, Turkey \\ ${ }^{2}$ Cumhuriyet University Faculty of Medicine, Department of Child and Adolescent Psychiatry, Sivas, Turkey \\ Received: 13 November 2020, Accepted: 09 December 2020, Published online: 31 December 2020 \\ (C) Ordu University Institute of Health Sciences, Turkey, 2020
}

\begin{abstract}
Objective: Although anorexia nervosa (AN) has been associated with increased suicidal attempts, clinical variables that that influence this relationship still remains to be elucidated. The aim of this study is to examine the frequency of suicide attempts in females with AN, and the general psychopathology and associated clinical variables in patients with anorexia nervosa who do and do not attempt suicide.

Methods: In this study, archive files and reports of 43 females with AN admitted to the department of child and adolescent psychiatry between 2015 and 2020 were analyzed retrospectively. Participants' sociodemographic variables (age, peer relationships, psychiatric comorbidity, abuse history, history of suicidal thought and/or plan, family history of $\mathrm{AN}$, etc.) and clinical (subtypes of $\mathrm{AN}$, clinical features, response to treatment, the presence of suicidal ideation/attempts, etc.) characteristics were investigated. Also, the Children's Depression Inventory (CDI) and the State-Trait Anxiety Inventory (STAI) were used.

Results: Our results revealed that $18.8 \%$ of patients with restricting AN, $81.3 \%$ of patients with purging or binging/purging $\mathrm{AN}$, and $37.2 \%$ of the entire sample had at least one suicidal attempt, and $39.5 \%$ of the patients engaged in nonsuicidal self-injury behaviors. Further, binge-eating/purging AN type, more severe forms of AN, treatment resistance and poor insight or lack of insight, the presence of physical and emotional abuse, domestic violence, and the existence of psychiatric comorbidity markedly elevated the risk of suicide. Conclusion: The present study shows that patients with AN should be investigated not only for problems with eating and weight but also for possible non-suicidal self-harm behaviors, suicidal attempts.
\end{abstract}

Key words: Anorexia nervosa, suicide, suicide attempt, adolescent, eating disorders.

Suggested Citation: Ucuz I, Uzun Cicek A. Evaluation of the frequency of suicidal thoughts and attempts and related factors in female adolescents with Anorexia Nervosa. Middle Black Sea Journal of Health Science, 2020; 6(3):305-315.

Address for correspondence/reprints:

DOI: $\quad 10.19127 / \mathrm{mbsjohs} .825642$

İlnur Ucuz

Telephone number: +90 (507) 2383095

ORCID-ID 0000-0003-1986-4688

E-mail: ilknur_27@yahoo.com 


\section{Introduction}

Eating disorders are serious diseases causing bodily and psychosocial dysfunctions and may sometimes result in death (Smith et al. 2019). Anorexia Nervosa (AN), which is a form of eating disorder, is defined with the DSM-5 (Diagnostic and Statistical Manual of Mental Health, Fifth Edition) criteria as having low body weight, fear of gaining weight, and doing behaviors that prevent weight-gain by limiting the energy received compared to body requirements (American psychiatric association 2013). Although the lifetime prevalence in adolescent girls is between $0.3 \%$ and $2.6 \%$, it is stated in studies that it rates from $0.1 \%$ to $0.3 \%$ in boys (Swanson et al. 2011, Smink et al. 2014, Keski-Rahkonen and Silén 2019).

Suicide is a devastating act of death that is committed by individuals with the intention of open or implicit death. When this self-harming behavior does not result in death, it is called a suicide attempt (Bridge et al. 2006). According to the World Health Organization, suicide ranks the second among the causes of death among young people between the ages of 10 and 24, and suicide attempts are 20 times more common than completed suicide (World Health Organization). In a metaanalysis study evaluating the suicide rates and causes in psychiatric disorders, it was emphasized that suicide rates were higher in all psychiatric diseases than in normal population; and that borderline personality disorder, anorexia nervosa, depression and bipolar disorder had the highest risk of suicide (Chesney et al. 2014).

Anorexia Nervosa has a high risk of mortality in adolescents who are between the ages of 15 and 24 (Hoang et al. 2014). The high mortality rate in patients with anorexia was previously associated with to hunger and secondary complications of hunger; however, recent studies show that deaths associated with suicide contribute to this high rate (Franko et al. 2004). According to a meta-analysis of Keshaviah et al., individuals with AN have an 18.1 times higher risk of mortality from suicide than the 15- to 34-yearold female population (Keshaviah et al. 2014). In this study, the purpose was to evaluate the frequency of suicidal thoughts/attempts and associated sociodemographic features and clinical factors in female adolescents with AN diagnosis in the light of the relevant literature. In this way, it was also targeted to identify the risk factors for suicide in AN.

\section{Methods}

\section{Study Design and Sampling Selection}

The study was designed in retrospective design, and received local ethics committee approval (No: 2020/1129), including the data of 43 patients who were followed up and treated with AN in line with the DSM-5 criteria in Department of Child and Adolescent Psychiatry of Inonu University between 2015 and 2020. The sociodemographic characteristics, history of abuse (all of the cases with a history of sexual abuse had forensic reports), subset of the disease, clinical characteristics, response to treatment, whether there was suicidal thoughts/attempts, the number/method of suicide attempts, peer relationships, the presence of comorbid psychiatric conditions, family history of $\mathrm{AN}$, family history of suicide, the Children's Depression Inventory (CDI) and the State-Trait Anxiety Inventory of the patients were evaluated in detail. Patients with incomplete data were excluded from the study.

Children's Depression Inventory (CDI): It was developed by Kovacs in 1992 to determine the severity of depression in children and in young people (Kovacs 1981) and was adapted into Turkish by Öy in 1990 (Öy 1991). CDI is a self-assessment scale and is filled by reading to children or reading to the child by him/herself. Based on a total of 27 items, responses are given scores ranging from $0-2$; and on 19 , the highest score that may be received is 54 , and higher scores show a higher level of depression.

State-Trait Anxiety Inventory for Children (STAI-C): The scale was developed by Spielberg et al., and has two separate subgroups as the state anxiety scale and trait anxiety scale each of which consists of 20 items (Gaudry et al. 1975). The scale was adapted into Turkish by Ozusta (1995). In trait anxiety scale, the child is often asked to rate how s/he feels, while in state anxiety scale s/he is asked to assess how s/he feels in that moment.

\section{Statistical Analysis}

IBM SPSS Statistics version 23 (IBM Corp., Armonk, NY, USA) was used for statistical analysis. Compliance of the data to normal distribution was determined by the one-sample Kolmogorov-Smirnov test. The numerical and categorical data were presented as number (n), median (min-max), percentage (\%), and mean \pm standard deviation (SD) whenever appropriate. The Chi-square Test and Mann-Whitney-U-Test were used to for statistical 
analysis. Also, to evaluate the predictor value of some main clinical variables on the frequency of suicidality, the Binary Logistic Regression Analysis (stepwise) were performed. $\mathrm{p}<0.05$ was considered statistically significant.

\section{Results}

\section{Demographic characteristics of the sample}

The study population consisted of forty-three adolescent girls with AN between 12 and 17 years of age (mean age: $15.16 \pm 1.17$ years). Twenty-seven (62.8) of the participants were from the urban area and $21(48.8 \%)$ were from a low economic level. Most of the cases $(n=30,69.8 \%)$ had problematic or poor peer relationships. $41.9 \% \quad(n=18)$ of the participants were smoking, $9.3 \%(\mathrm{n}=4)$ were using alcohol/substance. Sixteen participants (37.2\%) reported physical abuse, seven participants (16.3\%) sexual abuse, and twenty-two participants (51.2\%) emotional abuse. Domestic violence was present in 22 cases $(51.2 \%), 6(14 \%)$ of whom were only emotional abuse, while $16(37.2 \%)$ were both physical and emotional abuse. Regarding the psychiatric comorbidity, more than half of the participants $(n=27,62.8 \%)$ were diagnosed with at least one psychiatric comorbid disorder. With respect to their primary diagnoses, the most common diagnosis was depression $(n=13, \% 30.2)$, this was followed obsessive-compulsive disorder $(\mathrm{n}=7$, $\% 16.3)$, post-traumatic stress disorder $(\mathrm{n}=5, \% 11.6)$ and disruptive behavior disorders $(\mathrm{n}=2, \% 4.7)$ followed. None of the patients had a chronic disease. $23.3 \%(\mathrm{n}=10)$ of the patients had a positive family history of suicide and $20.9 \%(n=9)$ had a positive AN family history. The sociodemographic and familial characteristics of the participants are shown in Table 1.

Table 1. Demographic characteristics of the sample

\begin{tabular}{lll}
\hline Variables & Number (n) & Percentage (\%) \\
\hline Place of residence & 27 & 62.8 \\
Urban & 16 & 37.2 \\
Rural & & \\
\hline Family income level & 21 & 48.8 \\
The minimum wage/less than minimum wage & 51.2 \\
Above the minimum wage & 22 & \\
\hline Peer relationships & 13 & 30.2 \\
Good or average & 30 & 69.8 \\
Problematic or poor & 18 & 41.9 \\
\hline Smoking & 4 & 9.3 \\
\hline Use of alcohol/substance & 16 & 37.2 \\
\hline Physical abuse & 7 & 16.3 \\
\hline Sexual abuse & 22 & 51.2 \\
\hline Emotional abuse & & \\
\hline Domestic violence & 21 & 48.8 \\
No & 6 & 14 \\
Emotional only & 16 & 37.2 \\
Physical and emotional & 27 & 62.8 \\
\hline Psychiatric comorbidity & & \\
\hline Comorbid psychiatric disorders & 13 & 30.2 \\
Depressive disorders & 5 & 11.6 \\
Post-traumatic stress disorder & 7 & 16.3 \\
Obsessive-compulsive disorder & 2 & 4.7 \\
Disruptive behavior disorders & 10 & 23.3 \\
\hline Family history of suicide & 9 & 20.9 \\
\hline Family history of anorexia nervosa &
\end{tabular}

\section{Clinical features of anorexia nervosa}

The mean age of onset of anorexia was $13.67 \pm 0.96$ years. Participants' mean "body mass index" value was $15.89 \pm 0.62$. $62.8 \%(n=27)$ of the patients were diagnosed with restricting type AN, 37.2\% ( $n=16)$ binge-eating/purging type AN. The severity of AN was distributed as mild $(n=18,41.9 \%)$, moderate $(n=14,32.6 \%)$, severe $(n=8,18.6 \%)$ and extreme 


\section{Suicidal Attempts in Anorexia Nervosa}

$(\mathrm{n}=3,7 \%)$. Regarding the clinical features of anorexia, while 18 patients (41.9\%) had self-induced vomiting, 8 patients (18.6) had amenorrhea. 14 patients $(32.6 \%)$ were doing exercise excessively. Binge eating was present in 18 patients $(41.9 \%)$. There were no patients who misused laxatives, diuretics, or enemas. With regard to the motives of $\mathrm{AN}, 27$ patients $(62.8 \%)$ reported the "trying to look like their friends" as the factor that triggered their symptoms, while sixteen patients (37.2\%) defined the "feedback about being overweight" as the reason. Twenty patients (60.5\%) had previously received treatment for $\mathrm{AN}$ or were under treatment, and 21 patients $(48.8 \%)$ had treatment resistance and poor insight or lack of insight. The clinical features of anorexia are described in Table 2.

Table 2. Clinical features of anorexia nervosa

\begin{tabular}{lll}
\hline & Number (n) & Percentage (\%) \\
\hline Anorexia nervosa subtypes & & \\
Restricting type & 27 & 62.8 \\
Binge-eating/purging type & 16 & 37.2 \\
\hline Severity of AN & & \\
Mild & 18 & 41.9 \\
Moderate & 14 & 32.6 \\
Severe & 8 & 18.6 \\
Extreme & 3 & 7 \\
\hline Self-induced vomiting & 18 & 41.9 \\
\hline Absence of menstruation & 8 & 18.6 \\
\hline Exercising excessively & 14 & 32.6 \\
\hline Binge eating & 15 & 34.9 \\
\hline Motives of AN & & \\
Trying to look like friends & 27 & 62.8 \\
Feedback about being overweight & 16 & 37.2 \\
\hline Previous treatment for AN (in patient) & 26 & 60.5 \\
\hline Treatment resistance and poor insight or lack of insight & 21 & 48.8
\end{tabular}

Abbreviations: AN, anorexia nervosa

Evaluation of nonsuicidal self-harm behaviors, and suicidal thought, plan, and attempts

Nonsuicidal self-harm behaviors were detected in $39.5 \%(n=17)$ of the participants. Self-cutting $(n=17$, $39.5 \%)$, self-burning $(\mathrm{n}=9,20.9 \%)$ and skin damage by other methods $(n=9,20.9 \%)$ were determined as the nonsuicidal self-harm methods. While the frequency of suicidal thought and/or plan before AN were $53.5 \%(n=23)$, the frequency of suicidal attempt was $34.9 \%(n=15)$ before AN. As for method(s) of suicidal thought, plan or attempts before $\mathrm{AN}$, drug intake in 15 patients $(34.9 \%)$, drug intake+use of other methods in 7 patients (16.3\%) and jumping from a height in 1 patient $(2.3 \%)$ were found. The frequency of suicidal thought and/or plan after AN was $58.1 \%(\mathrm{n}=25)$, while the frequency of suicidal attempt after AN was $37.2 \%(n=16)$. Table 3 displays the data on features of nonsuicidal self-harm behaviors, and suicidal thought, plan, and attempts. 
Table 3. Features of nonsuicidal self-harm behaviors, and suicidal thought, plan, and attempts

\begin{tabular}{lll}
\hline & Number (n) & $\begin{array}{l}\text { Percentage } \\
(\%)\end{array}$ \\
\hline Nonsuicidal self-harm behaviors & 17 & 39.5 \\
\hline Methods of nonsuicidal self-harm & 17 & 39.5 \\
Self-cutting & 9 & 20.9 \\
Self-burning & 9 & 20.9 \\
Skin damage by other methods & 23 & 53.5 \\
\hline Suicidal thought and/or plan before AN & 15 & 34.9 \\
\hline Suicidal attempt(s) before AN & 15 & \\
\hline Method(s) of suicidal thought, plan or attempts before AN & 7 & 34.9 \\
Drug intake & 1 & 16.3 \\
Drug intake + Other method(s) & 25 & 2.3 \\
Jumping from height & 16 & 58.1 \\
\hline Suicidal thought and/or plan after AN & & 37.2 \\
\hline Suicidal attempt(s) after AN & 9 & \\
\hline Method(s) of suicidal thought, plan or attempts after AN & 9 & 20.9 \\
Drug intake & 7 & 20.9 \\
Drug intake + Other method(s) & & 16.3 \\
Jumping from height
\end{tabular}

Abbreviations: AN, anorexia nervosa

Comparison of clinical variables of patients with and without suicidal attempt(s) after AN

The mean age of those who had attempted suicidal attempts after AN was significantly higher than those who did not ( $p$ 0.004), but the mean age of onset of AN did not reach a significant value $(\mathrm{p}=0.566)$. AN subtype differed significantly between the two groups ( $<<0.001)$, accordingly, those who had suicidal attempt(s) had a higher rate of binge-eating/purging AN type than those who did not $(81.3 \%$ vs. 11.1 , respectively). The two groups also significantly differed in terms of the severity of AN ( $p<0.001)$; all of those who had attempted suicide had moderate or other serious types, this rate was $33.3 \%$ in those who did not. In contrast, average body mass index scores were not different between the two groups $(\mathrm{p}=0.241)$. Treatment resistance and poor insight or lack of insight rates of those who had attempted suicide after AN were significantly higher than those who did not attempt suicide after AN $(93.8 \%$ vs. $22.6 \%$, respectively, $\mathrm{p}<0.001$ ) (Table 4 ).

While psychiatric comorbidity was present in all those who had attempted suicide, this rate was $40.7 \%$ in those who did not, and the difference was statistically significant $(\mathrm{p}<0.001)$. Smoking rates (93.8\% vs. $11.1 \%$, respectively, p <0.001) and alcohol-substance use rates $(25 \%$ vs. $0 \%$, respectively, $\mathrm{p}=0.015$ ) were significantly higher in those who had attempted suicide compared to those who did not. In terms of the frequency of non-suicidal self-harm behaviors, a significant difference was observed between the groups ( $\mathrm{p}<0.001)$, accordingly, while all of those who had attempted suicide after AN had previously engaged in self-injurious behavior, only 1 patient $(3.7 \%)$ among those who did not attempt suicide had previously engaged in selfinjurious behavior. The rates of suicidal thought and/or plan before AN $(100 \%$ vs. $25.9 \%$, respectively), suicidal attempt(s) before AN (100\% vs. $0 \%$, respectively), and suicidal thought and/or plan after AN (100\% vs. $33.3 \%$, respectively) of those who had attempted suicide after AN were significantly higher than those of those who did not (all p-values $<0.001$ ). Moreover, our findings showed that the positive family history of suicide was significantly higher in those who had attempted suicide after AN than those who did not $(\mathrm{p}=0.001)$, whereas the family history of AN did not differ $(\mathrm{p}=0.06)$ (Table 4).

The frequency of physical abuse $(68.8 \%$ vs. $18.5 \%$, respectively, $\mathrm{p}=0.001$ ), emotional abuse $(87.5 \%$ vs. $29.6 \%$, respectively, $\mathrm{p}<0.001)$, and domestic violence $(87.5 \%$ vs. $29.6 \%$, respectively, $\mathrm{p}<0.001)$ among those who had attempted suicide were significantly higher compared to those who did not. However, there was no significant difference between the two groups in terms of sexual abuse $(\mathrm{p}=0.082)$. Regarding peer relationships, it was observed that those who had attempted suicide had more problematic or poorer peer relationships than those who did not, and the difference was statistically 
significant $(87.5 \%$ vs. $55.6 \%$, respectively, $\mathrm{p}=0.031)$ (Table 4).

As for the scale scores, the mean CDI scores (40.0 \pm 5.35 vs. $28.59 \pm 7.04$, respectively, $\mathrm{p}<0.001)$ and the mean STAI-trait ( $33.62 \pm 6.31$ vs. $30.41 \pm 6.30$, respectively, $\mathrm{p}=0.029$ ) scores of those who had attempted suicide were significantly higher than those of those who did not. However, the mean STAI-state scores were not different between groups $(\mathrm{p}=0.087)$. The comparisons of clinical variables of patients with and without suicidal attempt(s) after AN is given in Table 4.

Table 4. Clinical variables of patients with and without suicidal attempt(s) after AN. Data were given as mean \pm standard deviation or number (percent \%)

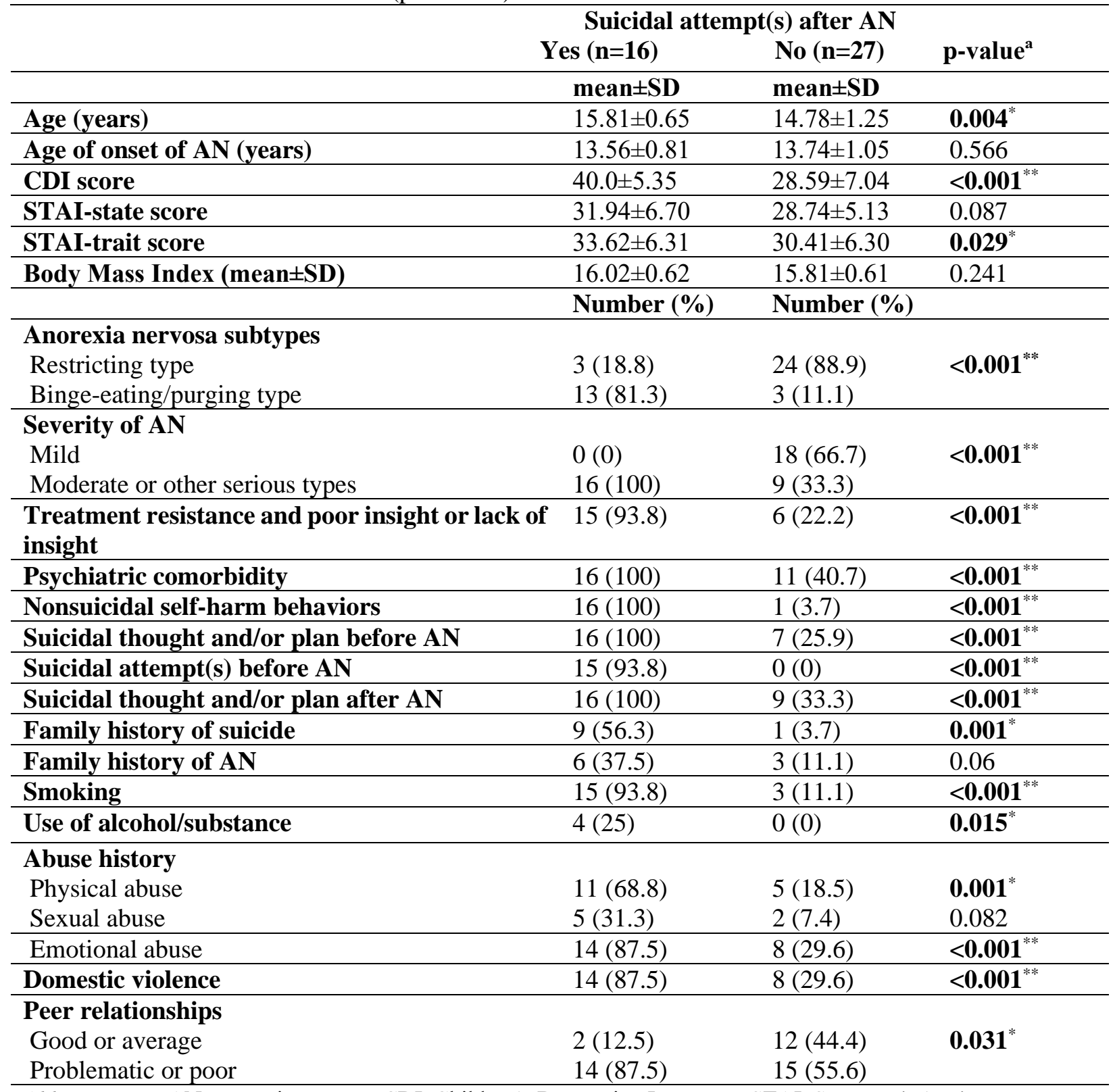

Abbreviations: AN, anorexia nervosa, CDI, Children's Depression Inventory, STAI, State-Trait Anxiety Inventory.

${ }^{a}$ The chi-square test and Fisher's exact test (as appropriate) for categorical variables and the Mann-Whitney U test for continuous variables were used to test group differences.

$*: \mathrm{p}<0.05,{ }^{* *}: \mathrm{p}<0.001$ 


\section{Logistic regression results for suicide attempt(s)}

In the multiple logistic regression analysis performed for suicide attempt after AN; among the sociodemographic variables, the patient's age, age at diagnosis of AN, family income level, place of residence, alcohol-substance use, domestic violence, physical/sexual/emotional abuse and neglect history did not affect suicide attempt risk ( $>>0.05$ for all variables). Regression analysis according to the mental illness of the patient and his family did not reveal the presence of comorbid psychiatric illness, a history of nonsuicidal self-harm behavior, a history of suicide or AN in the family, and a history of suicidal ideation/plan/attempt in the patient before AN on the risk of suicide attempt (for all variables. $p>0.05$ ). When analyzed in terms of AN properties; the effect of vomiting-amenorrhea, exercising, condition that causes AN and treatment resistance (low insight) on suicide attempt risk was not determined ( $p>0.05$ for all values). The binge-eating/purging subtype of AN increased the risk of suicide attempt 32 times $(\mathrm{p}=0.006)$ compared to the restrictive subtype, and the group with severe AN increased 16 times $(\mathrm{p}=0.011)$ compared to mild and moderate severity. In regression analysis based on scale scores, while STAI-state and STAI-trait scale scores did not have an increasing effect on suicide attempt risk, a 1-unit increase in depression scale score increased the risk of suicide attempt 1.2 times ( $<<0.001)$. The results of binary logistic regression analysis (stepwise) for suicide attempt were given in Table 5 .

Table 5. Results of Binary logistic regression analysis (stepwise) for suicide attempt

\begin{tabular}{|c|c|c|c|c|c|}
\hline $\begin{array}{l}\text { Sociodemographic } \\
\text { variables }\end{array}$ & $\begin{array}{l}\text { Reference } \\
\text { category }\end{array}$ & $\begin{array}{l}\text { Other } \\
\text { category }\end{array}$ & Beta & OR (\%95 Cl) & p value \\
\hline Smoking & Yes & No & 4,689 & $108,768(8.242-1435,331)$ & $0,000 * *$ \\
\hline Physical abuse & Yes & No & & & 0,090 \\
\hline $\begin{array}{l}\text { Features of mental } \\
\text { status }\end{array}$ & $\begin{array}{l}\text { Reference } \\
\text { category }\end{array}$ & $\begin{array}{l}\text { Other } \\
\text { category }\end{array}$ & Beta & OR (\%95 Cl) & p value \\
\hline $\begin{array}{l}\text { Nonsuicidal self- } \\
\text { harm behaviors }\end{array}$ & Yes & No & & & 0.097 \\
\hline $\begin{array}{l}\text { Suicidal attempt(s) } \\
\text { before AN }\end{array}$ & Yes & No & & & 0.998 \\
\hline $\begin{array}{l}\text { Family history of } \\
\text { anorexia nervosa }\end{array}$ & Yes & No & & & 0.997 \\
\hline $\begin{array}{l}\text { Clinical features of } \\
\text { AN }\end{array}$ & $\begin{array}{l}\text { Reference } \\
\text { category }\end{array}$ & $\begin{array}{l}\text { Other } \\
\text { category }\end{array}$ & Beta & OR (\%95 Cl) & p value \\
\hline Severity of AN & Severe-extreme & $\begin{array}{l}\text { Mild- } \\
\text { Moderate }\end{array}$ & 2.781 & $16,141(1.895-137.451)$ & 0.011* \\
\hline $\begin{array}{l}\text { Anorexia nervosa } \\
\text { subtypes }\end{array}$ & $\begin{array}{l}\text { Binge- } \\
\text { eating/purging } \\
\text { type }\end{array}$ & $\begin{array}{l}\text { Restricting } \\
\text { type }\end{array}$ & 3.471 & $32.166(2.699-383.321)$ & $0.006 *$ \\
\hline Scale scores & $\begin{array}{l}\text { Reference } \\
\text { category }\end{array}$ & $\begin{array}{l}\text { Other } \\
\text { category }\end{array}$ & Beta & OR (\%95 Cl) & p value \\
\hline CDI & - & - & 0.248 & $1.282(1.112-1.506)$ & $\mathbf{0 . 0 0 0} * *$ \\
\hline
\end{tabular}

OR: Odds ratio, CI: Confidence interval, CDI: Child depression inventory, AN: Anorexia nervosa, STAI: State-Trait anxiety inventory for children, *: $\mathrm{p}<0.05, * * \mathrm{p}<0.001$

\section{Discussion}

In this study, we examined the frequency of nonsuicidal self-harm and suicide attempts in females with AN, and the general psychopathology and associated clinical variables in patients with AN who do and do not attempt suicide. Our results established that $\mathrm{AN}$ is associated with an increased likelihood of suicidality (a variable of varying severity from transient or persistent thoughts, plans to attempt), and depressive symptoms and symptoms of higher anxiety mediate this relationship. We determined that $39.5 \%$ of the patients engaged in nonsuicidal self- harm behaviors, and $37.2 \%$ attempted suicide at least once. Further, binge-eating/purging AN type, moderate or other serious AN forms, treatment resistance and poor insight or lack of insight, the presence of physical and emotional abuse, domestic violence, the existence of psychiatric comorbidity, and problematic or poor peer relationships elevated the risk of suicide.

Previous studies have reported that AN patients have elevated rates of suicide attempts compared to the general healthy population and suicide contributes to excessive mortality rates in AN patients (Pompili 
et al. 2006, Bulik et al. 2008, Forcano et al. 2009, Forcano et al. 2011, Crow et al. 2014, Wade et el. 2015). It has been found that a significant proportion of patients with eating disorders, and AN in particular, have suicidal suicidal ideation at any time during the course of their illness and lifetime. The frequency of suicidal thoughts is given at the rate of one quarter to one-third (Swanson et al. 2011, Carano et al. 2012). Although the prevalence of suicidal attempts varies significantly among studies due to methodological differences including study design, the severity of the disorder, and demographic characteristics such as age and population composition, regarding the suicidal attempts, almost similar rates were also determined (Franko and Keel 2006, Crow et al. 2014). It has been reported that the risk of suicide in patients with AN is 6-10 times higher than in the general population (Mendolicchio et al. 2014). A prior study has found a frequency of suicidal attempts in $8.65 \%$ of outpatients with restricting $\mathrm{AN}$ and in $25.0 \%$ outpatients of purging AN (Forcano et al. 2009). In another study, it was found that $20 \%$ of anorexic patients attempted suicide, and the rate of successful ones was $5 \%$ (Franko et al. 2004). We found a higher rate of suicidal attempts than this study reported. Our results revealed that $11.1 \%$ of patients with restricting AN, $81.3 \%$ of patients with purging or binging/purging $\mathrm{AN}$, and $37.2 \%$ of the entire sample had at least one suicidal attempt. Our high rates may be due to our sampling strategy because most of the cases in our sample had moderate or other severe forms and a significant portion of the participants were recruited from the inpatient service. In addition, it has been shown that $40.8 \%$ of adolescents with eating disorders exhibit self-harming behaviors (Peebles et al. 2011). According to the types of eating disorders, the prevalence of self-injurious behavior is $26-55.2 \%$ in bulimia nervosa, 27.8-68.1\% in purging or binging/purging $\mathrm{AN}$, and $13.6-42.1 \%$ in restricting AN (Svirko and Hawton, 2007). We also found similar rates of nonsuicidal self-harming behaviors in this population.

With regard to underlying mechanisms or dynamics, a number of theories have been proposed to explain the increased suicidality in individuals with eating disorders. In general, asserted theories have argued that eating disorders themselves bring about suicidal behavior or that they contribute to increased suicidality by they co-occur with other factors that give rise to suicidal behavior1. It is emphasized that AN and psychopathological conditions that lead to suicidal behavior share common genetic and environmental risk factors (Koutek et al. 2016).
Accordingly, some authors have suggested that high comorbidity rates with other psychiatric disorders related to increased suicidality may make the patients more prone to suicide (Blinder et al. 2006). In parallel with this, it has been shown that the risk of suicidal attempts increases in the presence of comorbidity with conditions such as mood disorders, posttraumatic stress disorder, various impulse-control disturbances, and substance use, each of which is an independent risk factor for suicide (Franko et al. 2004, Bulik et al. 2008, Davico et al. 2019). There have even been reports that eating disorders are not substantially increasing suicidal propensity after adjusting for comorbidities (Forrest et al. 2016). In our study, the rate of psychiatric comorbidity was quite high with $62.8 \%$, and the most common diagnoses were disorders associated with a great risk of suicide such as depression, PTSD and OCD (Auerbach et al. 2015, Chou et al. 2020, Agne et al. 2020). Moreover, we found that psychiatric comorbidity is very strongly associated with suicidal attempts. In addition, we found that alcoholsubstance use, and especially smoking, are important risk factors for suicidal attempts among individuals with AN. Our results support the literature information (Franko et al. 2004, Auerbach et al. 2015, Lian et al. 2017, Chou et al. 2020, Agne et al. 2020). Eating disorders show comorbidity with $40-50 \%$ depression, $60-70 \%$ anxiety disorders, as well as other psychological disorders such as alcoholsubstance abuse and personality disorders (Swanson et al. 2011). Studies have also found that individuals with AN have a high risk of depression and that that suicide attempts in these patients are modified by depression (Favaro and Santonastaso et al. 1997, Koutek et al. 2016, Lian et al. 2017).

Apart from psychopathology, studies have indicated that purging behavior (Favaro and Santonastaso 1997, Franko and Keel 2006), low BMI (Favaro and Santonastaso 1997), earlier onset (Franko et al. 2004), longer disease duration (Favaro and Santonastaso 1997, Stein et al. 2004), history of physical and/or sexual abuse (Favaro and Santonastaso 1997), and worse prognosis (Steinhausen 2009) increase the risk of suicidality in patients diagnosed with AN. We could not assess the prognosis due to the design of our study, but all other clinical variables except BMI were associated with increased suicidality rates. Furthermore, we also detected that positive family history of suicide, a history of nonsuicidal self-harm behaviors, and the presence of suicidal thoughts, plans, and/or attempts before AN increase the risk of subsequent suicide. 
Although evidence is limited to a few studies that controlled for whether there is a difference in the incidences of suicidal attempts of two AN subtypes, research shows that patients who suffered from restricting AN have lower percentages of suicidal attempts (Favaro and Santonastaso 1997, Franko et al. 2004, Youssef et al. 2004). Similarly, we have also found higher frequency of suicide attempts in purging or binging/purging AN. Our results are consistent with the results of other studies showing that the presence of purging symptoms is more common in patients who have attempted suicide (Franko et al. 2004, Forcano et al. 2011). This finding has been attributed to the fact that "purging" behaviors are associated with greater psychopathology and impulsivity, and poorer emotional regulation, especially anger (Krug et al. 2008) each of which may increase the suicide risk.

More importantly, the outcomes of our study yielded that more severe forms of AN and rates of treatment resistance and poor insight or lack of insight are more frequent in cases who attempted suicide, suggesting the dose-response relationship between the severity of AN and suicidal attempts. Our findings support data from a recent study showing that patients with more severe AN have an elevated risk of suicidality (Fennig and Hadas 2010; Lian et al. 2017).

Other striking findings of this study are that AN case who attempted suicide have more physical and emotional abuse and domestic violence experiences and more problems in peer relationships. The most likely explanation for these findings may be that such stressful life events indirectly predispose to disorders that are robustly associated with suicidality, such as depression and post-traumatic stress disorder. However, there is insufficient data on whether these variables are predictive values for suicidality in patients with AN (Favaro and Santonastaso 1997). Therefore, further studies are needed to determine whether these variables contribute to the increased risk of suicidality in this population.

This study was subject to several limitations. First, the design of our study was retrospective. Second, our sample lacked a control group and consisted of a female group with AN only, hence, our sample is not representative for all anorexic patients. Third, our sample size did not allow us to evaluate the relationship between some clinical variables and suicidality risk. These weaknesses occlude the generalization of our results. Therefore, future longitudinal studies with large sample size including both a healthy control group and a male group with
AN would be considerably beneficial to elucidate the existing knowledge further.

\section{Conclusion}

To summarize, the current study demonstrates that the severity, type, and certain clinical variables of AN affect the relationship between AN and suicidality, and comorbid psychopathological conditions, in particular, depressive symptoms and symptoms of higher anxiety, mediate this relationship. Also, our results suggest that clinicians should be particularly aware of the great risk of suicide if there are conditions including binge-eating/purging AN type, treatment resistance and poor insight or lack of insight, the presence of physical and emotional abuse, domestic violence, and problematic or poor peer relationships. If there are these adverse states, possible nonsuicidal self-harm behaviors, suicidal thoughts, plans, and/or attempts of these patients must be investigated.

Ethics Committee Approval: Ethics committee approval was received for this study from the Inonu University Faculty of Medicine Non-Interventional Clinical Research Ethics Committee (Decision No: 2020-1129).

Peer-review: Externally peer-reviewed.

Author Contributions: Concept- I. U., A. U. C.; Design- I. U., A. U. C.; Data Collection and Processing- I. U.; Literature Review- I. U., A. U. C.; Writing- I. U., A. U. C.; Critical Review- A. U. C.

Conflict of Interest: No conflict of interest was declared by the authors.

Financial Disclosure: The authors declared that this study hasn't received no financial support.

\section{References}

Agne NA, Tisott CG, Ballester P, Passos IC, Ferrão YA. Predictors of suicide attempt in patients with obsessive-compulsive disorder: an exploratory study with machine learning analysis. Psychol Med 2020; 1-11.

American psychiatric association. Diagnostic and statistical manual of mental disorders. 5th ed. Arlington, Virginia: American Psychiatric Publishing; 2013.

Auerbach RP, Millner AJ, Stewart JG, Esposito EC. Identifying differences between depressed adolescent suicide ideators and attempters. J Affect Disord 2015; 186: 127-133. 
Blinder BJ, Cumella EJ, Sanathara VA. Psychiatric comorbidities of female inpatients with eating disorders. Psychosom Med 2006; 68: 454-462.

Bridge JA, Goldstein TR, Brent DA. Adolescent suicide and suicidal behavior. J Child Psychol Psychiatry 2006; 47: 372-394.

Bulik CM, Thornton L, Pinheiro AP, Plotnicov K, Klump KL, Brandt H, et al. Suicide attempts in anorexia nervosa. Psychosom Med 2008; 70: 37883.

Carano A, De Berardis D, Campanella D, Serroni N, Ferri F, Di lorio G, et al. Alexithymia and suicide ideation in a sample of patients with binge eating disorder. J Psychiatr Pract 2012; 18: 5-11.

Chesney E, Goodwin GM, Fazel S. Risks of all-cause and suicide mortality in mental disorders:a metareview. World Psychiatry 2014; 13(2): 153-60.

Chou PH, Ito M, Horikoshi M. Associations between PTSD symptoms and suicide risk: A comparison of 4-factor and 7-factor models. J Psychiatr Res 2020; 129: 47-52.

Crow SJ, Swanson SA, Le Grange D, Feig EH, Merikangas KR. Suicidal behavior in adolescents and adults with bulimia nervosa. Compr Psychiatry 2014;55:1534-39.

Davico C, Amianto F, Gaiotti F, Lasorsa C, Peleso A, Bosia C, et al. Clinical and personality characteristics of adolescents with anorexia nervosa with or without non-suicidal selfinjurious behavior. Compr Psychiatry 2019; 94:152115.

Favaro A, Santonastaso P. Suicidality in eating disorders: clinical and psychological correlates. Acta Psychiatr Scand 1997; 95: 508-14.

Fennig S, Hadas A. Suicidal behavior and depression in adolescents with eating disorders. Nord J Psychiatry 2010; 64: 32-39.

Forcano L, Fernandez-Aranda F, Alvarez-Moya E, Bulik C, Granero R, Gratacos M, et al. Suicide attempts in bulimia nervosa: personality and psychopathological correlates. Eur Psychiatry 2009; 24: 91-7.

Forcano L, Alvarez E, Santamaría JJ, JimenezMurcia S, Granero R, Penelo E, et al. Suicide attempts in anorexia nervosa subtypes. Compr Psychiatry 2011; 52: 352-358.

Forrest LN, Bodell LP, Witte TK, Goodwin N, Barlett ML, Siegfried N, et al. Associations between eating disorder symptoms and suicidal ideation through thwarted belongingness and perceived burdensomeness among eating disorder patients. J Affect Disord 2016; 195: 127-135.
Franko DL, Keel PK, Dorer DJ, Blais MA, Delinsky SS, Eddy KT, et al. What predicts suicide attempts in women with eating disorders? Psychol Med 2004; 34: 843-53.

Franko DL, Keel PK. Suicidality in eating disorders: Occurrence, correlates, and clinical implications. Clin Psychol Rev 2006; 26: 769-82.

Gaudry E, Vagg P, Spielberger, CDJMBR. Validation of the state-trait distinction in anxiety research. Multivariate Behavioral Research 1975; 10: 331-341.

Hoang U, Goldacre M, James A. Mortality following hospital discharge with a diagnosis of eating disorder: National record linkage study, England, 2001-2009. Int J Eat Disord 2014; 47: 507-15.

Keshaviah A, Edkins K, Hastings ER, Krishna M, Franko DL, Herzog DB, et al. Re- examining premature mortality in anorexia nervosa: a metaanalysis redux. Compr Psychiatry 2014; 55: 177384.

Keski-Rahkonen A, Silén Y. Incidence and prevalence of eating disorders among children and adolescents. Hebebrand J, Herpetz-Dahlmann B, editors. Eating Disorders and Obesity in Children and Adolescents. Missouri: Elsevier; 2019.

Koutek J, Kocourkova J, Dudova I. Suicidal behavior and self-harm in girls with eating disorders. Neuropsychiatr Dis Treat 2016; 12: 787-93

Kovacs M. Rating scales to assess depression in schoolage children. Acta Paedopsychatr 1981; 46: 305-15.

Krug I, Bulik CM, Vall-Llovera ON, Granero R, Agüera Z, Villarejo C, et al. Anger expression in eating disorders: clinical, psychopathological and personality correlates. Psychiatry Res 2008; 161: 195-205.

Lian Q, Zuo X, Mao Y, et al. Anorexia nervosa, depression and suicidal thoughts among Chinese adolescents: a national school-based crosssectional study. Environ Health Prev Med 2017; 22:30.

Mendolicchio L, Maggio G, Fortunato F, Ragione LD. Update on eating disorders: epidemiology, mortality and comorbidity. Psychiatr Danub 2014; 26(Suppl 1): 85- 88.

Ozusta Ş. State-Trait Anxiety Inventory for Children: a study of realibility and validity. Turk Psikoloji Dergisi. 1995; 10: 32-44. (Turkish). 
Oy, B. Children's depression inventory: A study of validity and reliability, in Turkish. Turkish Journal of Psychiatry 1991; 2: 132-7. (Turkish)

Peebles R, Wilson JL, Lock JD. Self-injury in adolescents with eating disorders: correlates and provider bias. J Adolesc Health 2011; 48(3): 3103.

Pompili M, Girardi P, Tatarelli G, Ruberto A, Tatarelli R. Suicide and attempted suicide in eating disorders, obesity and weight-image concern. Eat Behav 2006; 7: 384- 94.

Smink FRE, Van Hoeken D, Oldehinkel AJ, Hoek HW. Prevalence and severity of DSM 5 eating disorders in a community cohort of adolescents. Int J Eat Disord 2014; 47: 610-9.

Smith AR, Velkoff EA, Ribeiro JD, Franklin J. Are Eating Disorders and Related Symptoms Risk Factors for Suicidal Thoughts and Behaviors? A Meta- analysis. Suicide Life Threat Behav 2019; 49: 221-239.

Stein D, Lilenfeld LR, Wildman PC, Marcus MD. Attempted suicide and self-injury in patients diagnosed with eating disorders. Compr Psychiatry 2004; 45: 447-51.

Steinhausen HC. Outcome of eating disorders. Child Adolesc Psychiatr Clin N Am 2009; 18: 225-42.

Svirko E, Hawton K. Self-injurious behavior and eating disorders: the extent and nature of the association. Suicide Life Threat Behav 2007; 37(4): 409-21.

Swanson SA, Crow SJ, Le Grange D, Swendsen J, Merikangas KR. Prevalence and Correlates of Eating Disorders in Adolescents: Results from the national comorbidity survey replication adolescent supplement. Arch Gen Psychiatry 2011; 68: 714-723.

Wade TD, Fairweather-Schmidt AK, Zhu G, Martin NG. Does shared genetic risk contribute to the cooccurrence of eating disorders and suicidality? Int J Eat Disord 2015; 48: 684 -91.

World Health Organization. Suicide prevention (SUPRE). Available from: https://www.who.int/healthtopics/suicide\#tab=tab_1

Youssef G, Plancherel B, Laget J, Corcos M, Flament MF, Halfon O. Personality trait risk factors for attempted suicide among young women with eating disorders. Eur Psychiatry 2004; 19: 131-9. 